\title{
EFEITO DE DOSES DE METRIBUZIN NO CRESCIMENTO E NA CONVERSÃO DA ENERGIA SOLAR EM PLANTAS DE SOJA (Glycine max (L.) MERRILL)
}

\author{
P.J. Silva Neto1 \\ N.F. Lopes M.A. Oliva 2 \\ J.F. Silva 3 \\ L.R. Ferreira 4 \\ 1 Eng $^{\mathrm{o}}$ Agr $^{\mathrm{o}}$, Pesquisador da CEPLAC - CP \\ $081-68370 \quad$ Altamira, PA \\ 2 Prof. Adjunto IV, Depto. Biologia Ve \\ getal-UFV - 36570 Viçosa, MG. \\ 3 Prof. Titular, Depto. Fitotecnia-UFV \\ 36570 Viçosa, MG \\ 4 Prof. Assistente I, Depto. Fitotec nia- \\ UFV - 36570 Viçosa, MG \\ Parte da tese de Mestrado apresenta da \\ pelo primeiro autor a UFV.
}

\section{RESUMO}

O crescimento e a eficiência na conversão da energia solar foram estudados em soja (Glycine $\max ($ L.) Merrill, cv. 'Uberaba'), cultivada em condições de campo, sob quatro doses de metribuzin $(0$, 0,35; 0,70 e 1,05 kg i.a. ha ${ }^{-1}$ ).

O valor máximo da conversão da energia solar foi de $0,75 \%$, para as plantas cultivadas na maior dose do herbicida. Os valores da con versão da energia solar média durante o ciclo da cultura foram 0,$32 ; 0,31 ; 0,32$ e $0,33 \%$. em ordem crescente de dose do metribuzin.

De modo geral, na fase vegetativa as plantas controle apresentaram valores inferiores em todos os valores de crescimento determinados, superando as tratadas com metribuzin somente na fase reprodutiva, mostrando que no período crítico de competição o dano causado pelas plantas daninhas é maior que a possível fitotoxicida de causada pelo metribuzin.

PALAVRAS-CHAVE: metribuzin, análise de crescimento, conversão de energia.

\section{SUMMARY}

EFFECT OF METRIBUZIN DOSES ON THE GROWTH AND SOLAR ENERGY CONVERSION IN SOYBEAN (Glycine $\max (L$.) MERRILL) PLANTS

Growth analysis and evaluation of solar energy conversion in soybean (Glycine max (L.) Merrill, Ube raba) unver field conditions and four doses of metribuzin $(0,0 ; 0,35 ; 0,70$ and $1,05 \mathrm{~kg}$ i.a.ha ${ }^{-1}$ ) were per formed in this study.

Maximum solar energy conversion was $0,75 \%$ for plants treated with $1,05 \mathrm{~kg}$ i.a.ha ${ }^{-1}$ metribuzin. 
The average of solar energy conversion through out the entire crop cycle were 0,32 , $0,31,0,32$ and $0,33 \%$ for the increasing metribuzin doses.

In general, the control showed lower figures for all grow th values studied than the treated during the vegetative phase. During

the reproductive phase, however, they surpassed those treated with metribuzin, showing that in the critical period of competition weeds were more harmful than the phytotoxicity produced by metribuzin.

KEYWORDS: metribuzin, growth analysis, ener gy conversion.

\section{INTRODUÇÃO}

O aumento da eficiência da utilização da energia solar, o maior período de enchimento de sementes e a maior concentração de assimilados nas sementes são fatores relevantes na maximização da produção de soja (21). No entanto, esses fatores dependem dos recursos do ambiente pelos quais as plantas daninhas competem com a cultura da soja. Características de crescimento, tais como índice de área foliar, taxa de produção de matéria seca, taxa de crescimento relativo e taxa assimilatória líquida são ótimos indicadores do efeito da competição entre as plantas daninhas e a soja $(7,14,15,16)$, além da produção final de grãos $(1,8,9)$.

Alguns herbicidas são utilizados de modo eficiente para reduzir essa competição. Todavia, trabalhos de Hardcastle $(10,11)$ mostraram que cultivares de soja respondem diferentemente quanto a tolerância ao herbicida metribuzin, cujo mecanismo de ação envolve inibição no processo de fotossíntese.
O presente estudo teve por objetivo analisar o efeito de doses de metribuzin no crescimento, na assimilação líquida e na conversão da energia solar da soja.

\section{MA TE RI AL E MÉ TO DO S}

O experimento foi conduzido, em Viçosa-MG, no período de dezembro de 1985 a maio de 1986, em condições de campo, num terreno que apresenta topografia plana, sendo o solo classificado como Podzólico Vermelho-Amarelo, com fertilida de média, textura argilosa e 2,9\% de matéria orgânica. A soja, cultivar 'Uberaba', foi semeada e, em préemergência, aplicado o metribuzin nas doses de 0,$0 ; 0,36 ; 070$ e 1,05 kg i.a.ha- ${ }^{1}$. As condições experimentais foram as descritas por Silva Neto et al. (s.d.).

$\mathrm{O}$ del ineamento experimental adotado foi o de blocos ao acaso, em parcelas subdivididas, com três repetições. Cada parcela representava uma época de coleta de material, realizada a intervalos regulares de catorze dias, a partir da emergência das plântulas, durante todo o ciclo da cul tura, perfazen do um total de 10 coletas. Cada parcela era formada de quat ro sub-parcelas, correspond entes às doses de metribuzin.

Em cada coleta, na área útil, foi realizado um acompanhamento dos estádios vegetativos (V) e reprodutivos da soja (R), segundo a escala de Fehr e Caviness (6), e, posteriormente, as plantas foram cortadas rente ao solo, com o sis tema radicular sendo extraído em blocos de terra e lavado sobre peneiras. A matéria fresca, tanto da parte aérea quanto das raízes, era determinada no próprio local. De cada subparcela, foram tomadas 
amostras constituídas de nove plantas, separadas em partes (raiz, caule, folhas, vagens e sementes). Para a obtenção do peso da matéria seca, o material vegetal foi colocado em estufa de ventilação for çada, à temperatura de $75^{\circ} \mathrm{C}$, onde permaneceu até peso constante. A área foliar foi determinada por meio de um medidor de área foliar portátil LI-COR, modelo LI3000 .

Os dados primários de matéria seca total $\left(\mathrm{W}_{\mathrm{t}}\right) \mathrm{e}$ área foliar (Af) foram submetidos ã anál ise de variância. Curvas logísticas decrescimento (18) foram ajustadas por meio de um programa interativo, para minimizar a variância residual. A equa ção logística empregada foi $\mathrm{W}_{\mathrm{t}}=\mathrm{W}_{\mathrm{m}} /\left(1+\mathrm{Be}^{\mathrm{Ct}}\right)$, sendo $\mathrm{W}_{\mathrm{m}}$ a estimativa assintótica do crescimento máximo; $\mathrm{B}$ e $\mathrm{C}$, constantes de ajustamento; e t, o tempo, em dias, $\mathrm{A}$ regressão curvilínea das medias de Af, acumulada em cada coleta, foi efetuada empregando polinômios ort ogonais. Procurou-se chegar ao pol inômio que melhor se ajustasse aos valores primários de Af, de acordo com Richards (18).

Para determinar os valores instantâneos da taxa de produção de matéria seca $\left(\mathrm{C}_{\mathrm{t}}\right)$ e da taxa de crescimento da área foliar $\left(\mathrm{C}_{\mathrm{A}}\right)$ foram empregadas as derivadas das equações ajustadas do peso da mate ria seca total $\left(\mathrm{W}_{\mathrm{t}}\right)$ e da área foliar $\left(\mathrm{A}_{\mathrm{f}}\right)$, em relação ao tempo(17, '18) .

Os valores instantâneos da taxa de crescimento relativo (Rw) e da taxa de crescimento relativo da área foliar (RA) foram calculados por meio das fórmulas: $\mathrm{R}_{\mathrm{w}}=\mathrm{Ct} / \mathrm{W}$ te $\mathrm{R}_{\mathrm{A}}=\mathrm{CA} / \mathrm{Af}$.

Para obtenção da taxa assimila tória liquida $\left(\mathrm{E}_{\mathrm{A}}\right)$ foram usados os valores instantâneos obtidos de $\mathrm{Ct}$ e Af, empregados na equa ção $\mathrm{E}_{\mathrm{A}}=\mathrm{Ct} / \mathrm{Af}$.
A razão da área foliar $\left(\mathrm{F}_{\mathrm{A}}\right)$, a razão de peso foliar $\left(\mathrm{F}_{\mathrm{w}}\right)$ e a área foliar especifica $\left(\mathrm{S}_{\mathrm{A}}\right)$ foram deter minadas a partir de valores instantâneos de $A_{f}, W_{f}$ e $W_{t}$, empregados nas equações $F_{A}=A f / W f ; F_{w}=W f / W_{t}$ e $S_{A}$ $=\mathrm{A}_{\mathrm{f}} / \mathrm{W}_{\mathrm{f}}$, de acordo com Rad ford (17).

Os valores instantâneos da eficiência de conversão da energia solar (0 foram determinados a partir da equação $\& \%=$ $\left(100 \mathrm{xC}_{\mathrm{t}} \mathrm{xE}\right.$.) $/ \mathrm{Ra}$, sendo $\mathrm{Ra}$ o valor médio diário da radiação solar total incidente, registrado nos cinco dias anteriores ao $\mathrm{C}_{t}$ correspondente. O valor calorífico (6) médio utilizado foi de 4460 cal.g- ${ }^{1}$, segundo determinações realizadas por Melges (13).

Os dados meteorológicos de radiação solar diária, de temperaturas máxima, média e mínima diárias, de precipitação diária e umidade relativa do ar, durante a condução do experimento, foram obtidos na Estação Climatológica Principal da Universidade Federal de Viçosa.

\section{RE SU LT AD OS E DI SC US SÃ O}

O crescimento da soja apresentou uma tendên cia sigmoidal, com três fases distintas: uma inicial, anterior à floração, quando o crescimento foi relativamente lento, indo do estádio VE até V8, aproximadamente; uma intermediária, em torno do final dos estádios vegetativos até o início da formação das sementes, quando o crescimento foi acelerado (V8 a R5); e uma final, quando ocorreu completo desenvolvimento das sementes e maturação, em que o acréscimo de matéria seca é muito lento e as curvas aproximam-se de um patamar (R5 a R8). Nota-se que as doses de me 


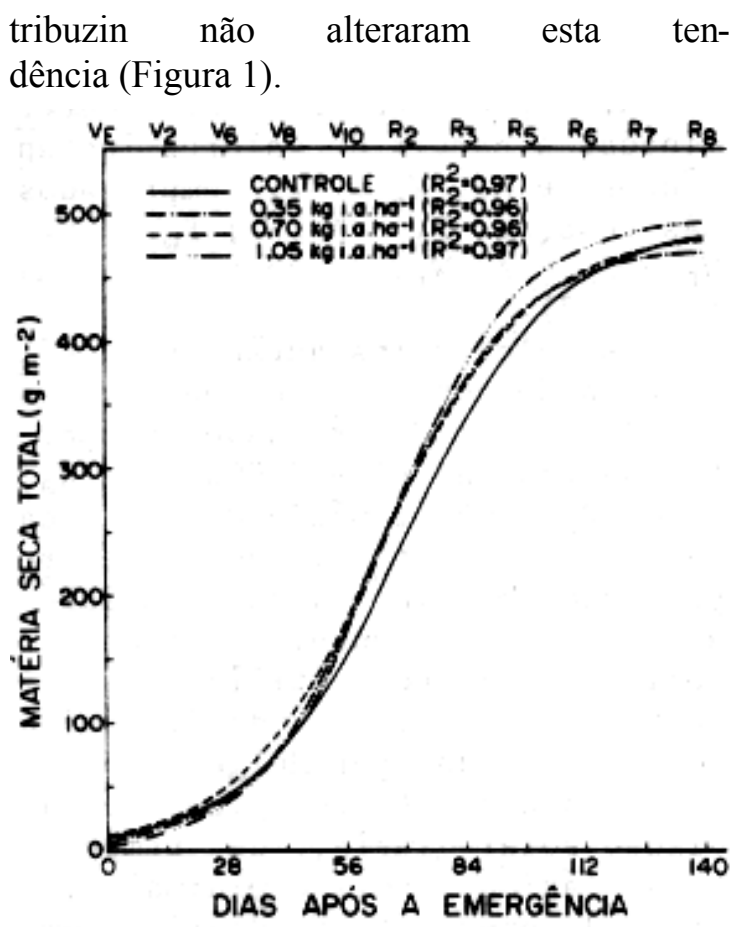

Figura 1. Acúmulo de matéria seca total em plantas de soja submetidas a quatro doses de metribuzin, sendo VE até R 8 estádios de desenvol vimento da soja.

Os valores do acúmulo de maté ria seca total $\mathrm{W}_{\mathrm{t}}$ foram praticamente semelhantes em todas as doses utilizadas, e as plantas submetidas à dose de $1,05 \mathrm{~kg}$ i.a. ha ${ }^{-\mathrm{T}}$ de metribuzin apresentaram maior es valores de $\mathrm{W}_{\mathrm{t}}$, a partir do estádio R3; no período entre os estádios $\mathrm{V}_{8}$ e $\mathrm{R}_{6}$, as plantas-controle apresentaram menor acúmulo de matéria seca em relação aos demais tratamentos.

A taxa de produção de matéria seca $\left(\mathrm{C}_{\mathrm{t}}\right)$ aumentou durante os primeiros 60 e 70 dias após a emergência das plântulas, chegando a uma taxa máxima e, a seguir, decrescendo rapidamen te (Figura 2).

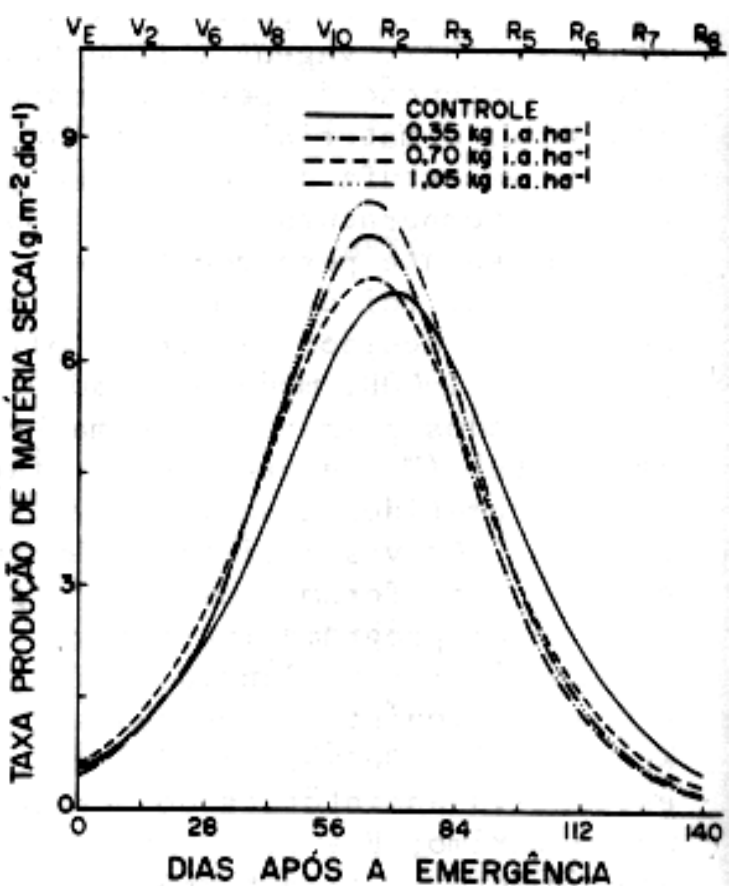

Figura 2. Taxa de produção de mate ria seca de plantas de soja submetidas a quatro doses de metribuzin, sendo VE até V8 os estádios de desenvolvimento da soja.

Verifica-se, inicialmente, que do estádio $\mathrm{V}_{\mathrm{E}}$ até $\mathrm{V}_{6}$ não houve variação nos tratamentos estudados. A partir do estádio $\mathrm{V}_{6}$ ate $\mathrm{R}_{3}$, as plantas submetidas às dosesde 0,$35 ; 0,70$ e $1,05 \mathrm{~kg}$ i.a.ha- ${ }^{1}$ de metribuzin começaram apresentar ma iores valores de $C_{t}$ em relação às plantascontrole. Isto, provavelmente, ocorreu em razão da falta de concorrência inicial das plantas daninhas com a cultura da soja, uma vez que o herbicida controlou a maioria das dicotiledôneas presentes na áre a experimental, ocasionando somente de leve a moderada clorose nas folhas de soja (23). Os valores máxi mos de $\mathrm{Ct}$ 
obtidos foram 6,$9 ; 7,7 ; 7,1$ e 8,1 g.m ${ }^{2}$.dia- ${ }^{1}$, em ordem crescente de dose de metribuzin. Esses valores ocorreram aos 70 e 63 dias após a emergência, para as plantas-controle e para os demais tratamentos, respectivamente. Valores de $C_{t}$ comparativos a este experimento foram obtidos por Melges(13) em cultivo de soja, cultivar 'UFV-2', crescidas à plena e a $70 \%$ da radiação solar incidente.

Os maiores valores de $C_{t}$ para as plantascontrole em relação aos demais tratamentos, na fase reprodutiva - $\left(\mathrm{R} 3\right.$ até $\left.\mathrm{R}_{8}\right)$, foram em virtude dos seus maiores valores de $\mathrm{E}_{\mathrm{A}}$ neste mesmo período (Figura 4).

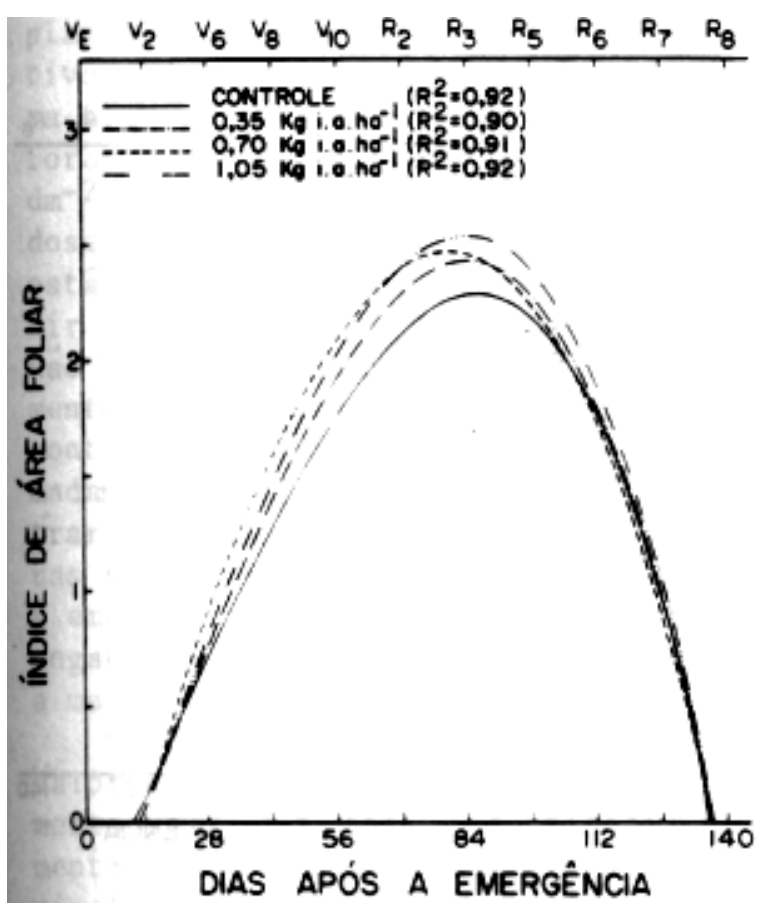

Figura 3. índice de área foliar de plantas de soja submeti das a quatro doses de me tribuzin, sendo VE até R8 os estádios de desenvolvimento da soja.
$\mathrm{O}$ efeito do metribuzin sobre o índice de área foliar (L) torna-se evidente a partir do estádio V6, quando as curvas das plantas subme tidas às doses de 0,$35 ; 0,70$ e1,05 kg i.a.ha- ${ }^{1}$ começaram a ultrapas sar a curva das plantas - controle (Figura 3) mostrando que não houve interferência direta (fitotoxicida de) e evidenciando que o herbicida nas doses util izadas neste experimento interferiu, de modo positivo, em L. Doses subletais de metribuzin, em cas a de vegetação, estimularam o aumento da área foliar (22). Em condições de campo, as doses utilizadas controlaram a maioria das dicotiledôneas, redu-

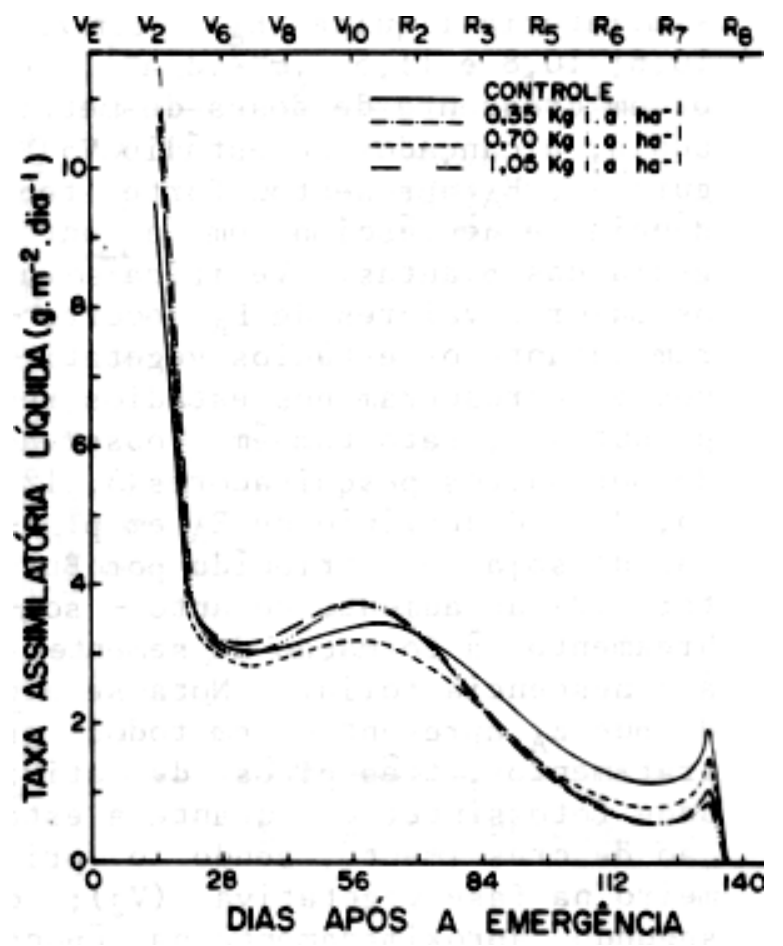

Figura 4. Taxa assimilatória líquida de plantas de soja sub metidas a quatro doses de metribuzin, sendo VE até R 8 os estádios de de senvolvime nto da soja. 
zindo a competição inicial com a soja (23). L máximo foram 2,$3 ; 2,4 ; 2,5$ e 2,6, em ordem crescente dedo se de metribuzin, atingidos em tor no de 84 dias após a emergência, nas doses de 0,$0 ; 0,35$ e $1,05 \mathrm{~kg}$ i.a.ha- ${ }^{1}$ de metribuzin, e de 77 dias, na dose de 0,70 , sendo semelhantes aos obtidos por Melges(13) e um pouco inferiores aos observados por outros autores (3, $5,9,15,24)$. De maneira geral, L aumentou durante o crescimento e o desenvolvimento vegetativo até que as plantas, em sua maioria, atingiram a fase reprodutiva, e, posteriormente, decresceu até a colheita.

Os valores máximos da taxa assimilatória líquida $\left(\mathrm{E}_{\mathrm{A}}\right)$ foram 7,$4 ; 10,6 ; 10,8$ e 11,5 g.m ${ }^{2}$.dia- ${ }^{1}$, em ordem crescente de doses de metribuzin, alcançados no estádio V2 (Figura 4). $E_{A}$ apresentou forte tendência de decréscimo com a ontogenia das plantas. Verifica-seque os maiores valores de $\mathrm{E}_{\mathrm{A}}$ ocorreram durante os estádios vegetativos e decresceram nos estádios reprodutivos, fato também observado por outros pesquisadores $(3,12,13,20)$. $\mathrm{O}$ declínio de $\mathrm{E}_{\mathrm{A}}$ em plantas de soja foi atribuído por Buttery (2) ao aumento do auto - sombreamento, à formação de sementes e à senescência foliar. Nota-se ainda que $\mathrm{E}_{\mathrm{A}}$ apresentou em todos os tratamentos, três picos de atividade fotossintética durante a estação de crescimento, sendo o primeiro na fase vegetativa $\left(\mathrm{V}_{2}\right)$; o segundo, aproximadamente na época do início da floração (R1); e o terceiro, durante o final do período de enchimento da vagem e maturação $\left(R_{7}\right.$ e $\left.R 8\right)$. Estes picos de $E_{A}$ verificados devem-se possivelmente, ao estímulo da fotossíntese causado pelo aumento do tamanho do dreno. Em soja, a elevação de $E_{A}$ verificada durante a época do enchimento das vagens foi interpretada como sendo um aumento da fotossíntese, em razão da mai or de manda de fotoassimilados para a rápida for mação de sementes $(4,12)$

Observa-se na Figura 5 que até o estádio V8, ap rox imadamente, as plantas tratadas com metribuzin apresentaram valores da taxa de crescimento de área foliar (CA) superiores aos das plantascontrole, mostrando que a competição pelas plantas daninhas até 40 dias após a emergência da soj a (23) provo cou enores taxas de $\mathrm{C}_{\mathrm{A}}$ do que a dose mais elevada do herbicida.

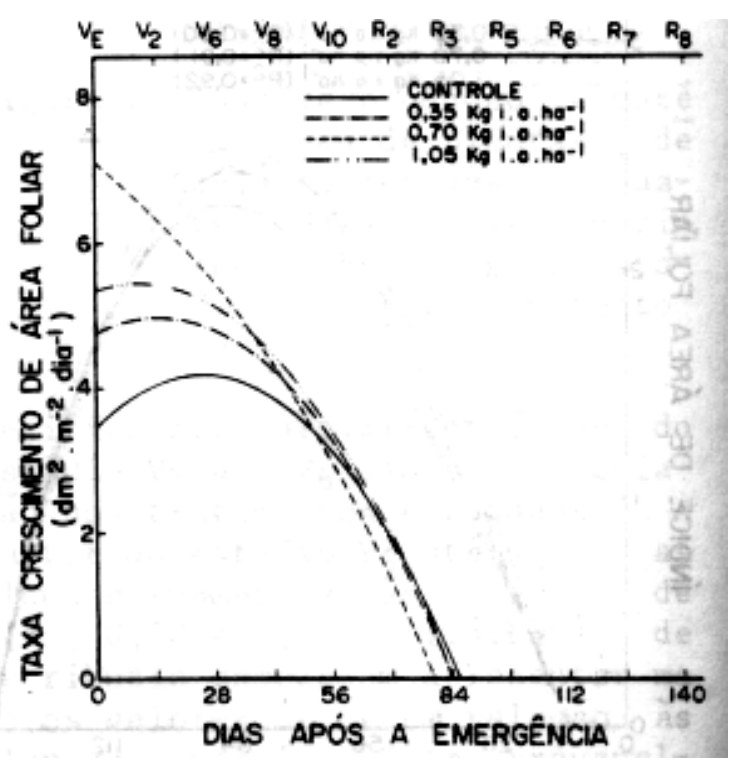

Figura 5. Taxa de crescimento de área foliar de plantas de soja submetidas a qua tro doses de metribuzin, sendo $V_{E}$ ate $R_{8}$ os estádi os de desenvolvimento da soja. 
Nota-se, também, que a partir do estádio V10 praticamente não houve diferenças em CA entre os tratamentos. Os valores máximos de CA foram 3,5; 4,7; 7,1 e 5,3 $\mathrm{dm}^{2}$. m-2. dia- ${ }^{1}$, em ordem crescente de dose de metribuzin. Verifica-se que $C_{A}$ vai diminuindo com a idade das plantas, atingindo valores negativos ainda no inicio da formação da vagem (estádio R3), indicando predominância da senescência foliar sobre o desenvolvimento de novas folhas.

Os valores máximos da taxa de crescimento relativo da área foliar (RA) foram alcançados nos primeiros estádios de desenvolvimento da soja, decrescendo, posteriormente, de acordo com a idade das plantas e atingindo valores negativos a partir do estádio R3 (Figura 6). As maiores taxas de RA foram 0,$36 ; 0,89 ; 1,03$ e $1,29 \mathrm{dm} 2$. dm ${ }^{2}$.dia- ${ }^{1}$, em ordem crescente de doses de metribuzin, observadas no estádio $\mathrm{V}_{2}$. Verifica-se que a partir de 28 dias após a emergência não houve diferença entre os trata mentos. As baixas taxas de $\mathrm{R}_{\mathrm{A}}$ encontradas até o estádio V6, aproximadamente, nas plantascontrole mos traram que a competição das invasoras com a cultura até 40 dias após a emergência (23) exerceu efeitos negativos em $\mathrm{R}_{\mathrm{A}}$, em comparação com a maior dose de metribuzin.

Na Figura 7 observa-se que os valores iniciais da taxa de cresci mento relativo $\left(\mathrm{R}_{\mathrm{W}}\right)$ são relativamente altos até o estádio V6,principalmente nas doses de 0,35 e 1,05 kg i.a.ha1 , sendo o menor valor observado nas plantas controle neste período em virtude da competição com as plantas daninhas. Os valores de $\mathrm{R}_{\mathrm{W}}$ decresceram rapidamente do estádio V6 ate R6, passando em seguida para uma fase de declínio mais lento até a maturação, pó- rem com valores sempre positivos. Verifica-se que após o estádio R2 as curvas passam a ser semelhan tes, não havendo praticamente di ferenças entre os tratamentos. Os valores máximos de $\mathrm{R}_{\mathrm{w}}$ foram de 55; 64,58 e 65 mg.g- ${ }^{-1}$.dia ${ }^{1}$, em ordem crescente de doses de metribuzin. Esses valores aproximam-se bastante dos obtidos por Melges (13) e Rocha et al. (19). Taxas máximas de 130 e 170 mg.g- ${ }^{1} \cdot \mathrm{dia}^{1}$ foram registradas por Köller et al. (12) e Scott e Batchelor (20), respectivamente.

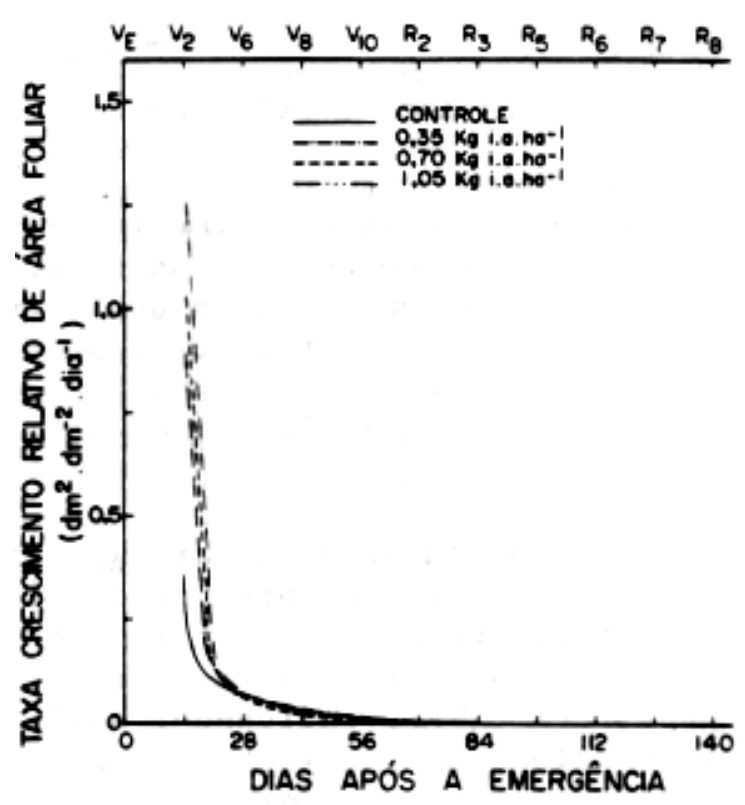

Figura 6. Taxa de crescimento relativo de área foliar de plantas de soja submetidas a quatro doses de me tribuzin, sendo VE até R 8 os estádios de desenvolvimento da soja. 


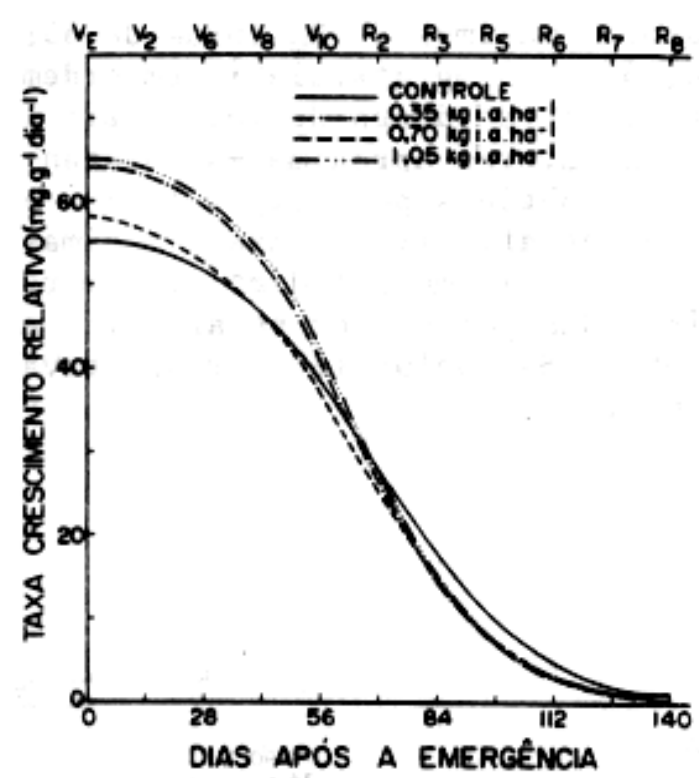

Figura 7. Taxa de crescimento relativo de plantas de soja submetidas a quatro do ses de metribuzin, sendo VE ate $R_{8}$ os estádios de desenvolvimento da soja.

A razão da área foliar $\left(\mathrm{F}_{\mathrm{A}}\right)$ (Figura 8) é um componente morfológico que representa a superfície assimilatória em relação a matéria seca total (Wt), indicando, assim, a transloc ação e a partição de assimilados entre as folhas e a plan ta toda. Observa-se que os valores de $\mathrm{F}_{\mathrm{A}}$ cresceram nos primeiros estádios de desenvolvimento da soja em todos os tra tamentos até, aproximadamente, 20 dias após a emergência, apresentando valores de FA semelhantes, Os valores máximos de $F_{A}$ ocorreram ao redor do estádio $\mathrm{V}_{6}$ ressaltando-se que as plantas submetidas a maior dose de me- tribuzin apresentaram FA superiores aos demais tratamentos. $\mathrm{F}_{\mathrm{A}}$ má ximo foi atingido no inicio do ciclo vegetativo, indicando que, primeiramente, o produto da fotossíntese foi convertido em folhas. A pós o estádio V6, os valores de FA começaram a declinar e, a partir de 50 dias após a emergência, tornaram-se semelhantes em todos os tratamentos, ate a colheita. Esse declínio de $\mathrm{F}_{\mathrm{A}}$ com a ontogenia das plantas indica que, progressivamente, menor quantidade de assimila:: dos é destinada à produção de folhas, mesmo quando $\mathrm{L}$ aumenta ate, aproximadamente, duas semanas após o início da floração (20).

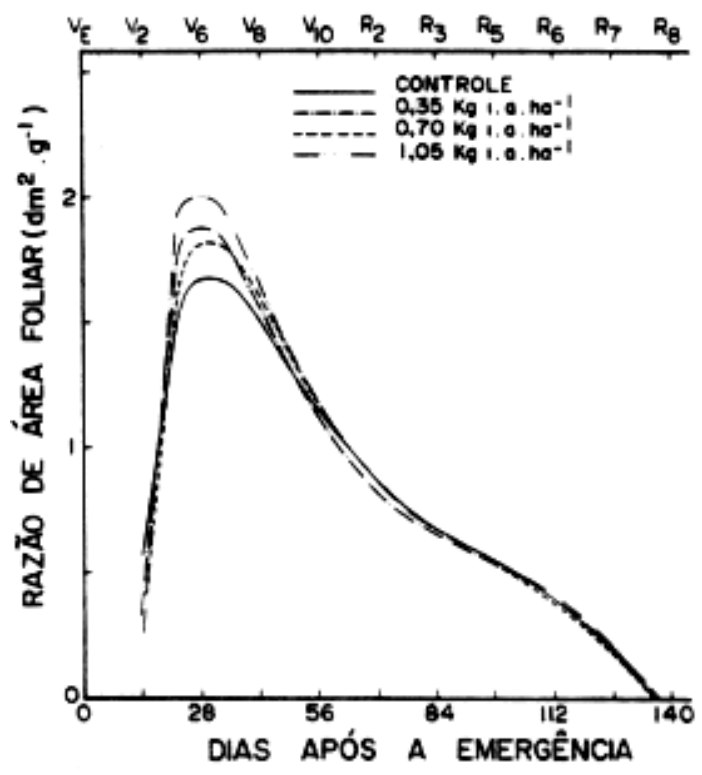

Figura 8, Razão de área foliar de plantas de soja submetidas a quatro doses de me tribuzin, se ndo VE até R8 os estádios de desenvolvi mento da soja. 


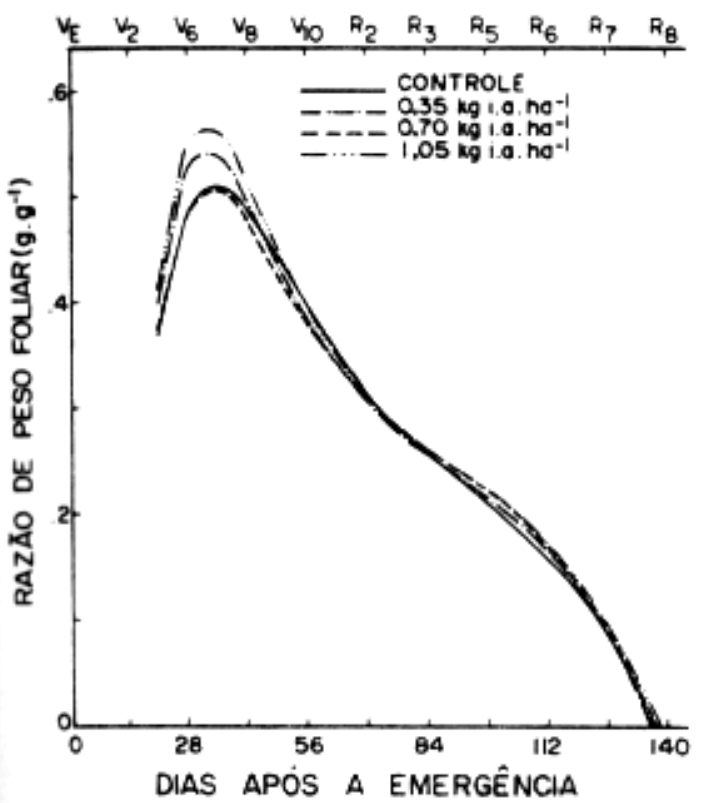

Fig. 9. Razão de peso foliar de plantas de soja submetidas a quatro doses de metribuzin, sendo VE ate R8 os estádios de desenvolvimento da soja.

$\mathrm{Na}$ Figura 9 observa-se que razão de peso foliar (Fw) apresentou valores crescentes até, aproximada mente, 35 dias após a emergência, ress altan dese que entre os estádios $\mathrm{V}_{6}$ e $\mathrm{V} 8$ as plantas cultivadas sob as doses de 0,35 e 1,05 i.a. $h^{-1}$ de metribuzin apresentaram os maiores valores. A partir do estádio de $\mathrm{F}_{\mathrm{W}}$ passaram a declinar, não apresentando diferenças entre os tratament os ate a colheita. $\mathrm{O}$ valor máximo de $\mathrm{F}_{\mathrm{w}}$ obtido nas plan tas sob a dose de $1,05 \mathrm{~kg}$ i.a. $\mathrm{ha}^{-1}$ e aproximado ao relatado por Clawson et al. (3) e Scott e Batchelor (20). As Figuras 8 e 9 mostram que tanto FA quanto $F_{w}$ apresentaram ten- dências semelhantes, e a competição inicial entre as plantas daninhas com a soja causou maiores na-nos do que a fit oto xicidade inicial do her bicida (23).

Os valores da área foliar especifica $\left(\mathrm{S}_{\mathrm{A}}\right)$ decresceram rapidamente do estádio V2 até V6, com ex ceçao das plantas submetidas à doses de $0,70 \mathrm{~kg}$ i.a.ha- ${ }^{1}$ de metribuzin, que tiveram os valores aumentados neste período(Figura 10). Após o estádio $\mathrm{V}_{8}$, as plantas mostraram a mesma tendência, não se verificando diferenças nos valores de SA, devido aos tratamentos. O decréscimo de $\mathrm{S}_{\mathrm{A}}$ durante os estádios de desenvolvimento da soja indica que as fol has não se expandem às mesmas taxas quando o crescimento progride (20). O valor máximo de $\mathrm{S}_{\mathrm{A}}$ obtido neste experimento foi de $6,4 \mathrm{dm}^{2} \cdot \mathrm{g}_{-}{ }^{1}$, nas plantas submetidas à dose de $0,35 \mathrm{~kg}$ i.a.ha ${ }^{-1}$ de metribuzin, no estádio V2. Em experimento de campo, Melges (13) obteve valores máximos de SA entre 7 e $12 \mathrm{dm}^{2} . \mathrm{g}^{-}$ 1 , sendo que no período de 42 a 126 dias após a emergência os seus valores de SA para o nível de radiação solar de 100\% foram semelhantes aos obtidos neste trabal ho.

As curvas de eficiência da conversão da energia solar (E) (Figura 11) mostram-se irregulares, com grandes variações de $E$, possívelmente em virtude das oscilações dos valores da radiação solar entre os dias. Observase que durante a fase vegetativa ate o estádio $\mathrm{R} 3$ as plantas-controle apresentaram valores de inferiores, superando as tratadas com metribuzin somente na fase reprodutiva ( $\mathrm{R} 3 \mathrm{e}$ R8), Contudo, os valores médios de $\xi$ for am iguais, ou seja: 0,$32 ; 0,31 ; 0,32$ e $0,33 \%$, em ordem crescente de doses de metribuzin. Ess es valores são semelhantes aos obtidos por Melges (13) 


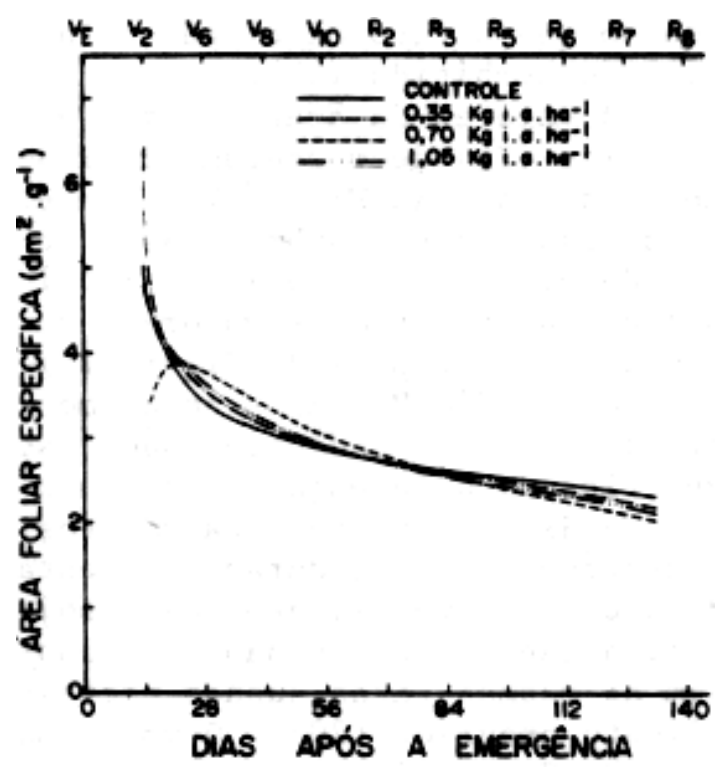

Figura 10. Área foliar específica de plantas de soja submetidas a quatro doses de metribuzin, sendo VE até R8 os estádios de desenvolvimento da soja.

em trabalho realizado com soja, cultivar 'UFV-2', sob o nível de $100 \%$ de radiação solar. O valor máximo de e, alcançado aos 60 dias após a emergência, foi de $0,75 \%$, nas plant as cultivad as sob a mai or dose do herbicida.

\section{LITEATURA CITADA}

1. Burnside, O.C. Soybean (Gluci ne max) growth as effected by weed removal cultivar, and row spacing. Weed Sci., 27: 562-565, 1979.

2. Buttery, B.R. An analysis of the growth of soybean as af

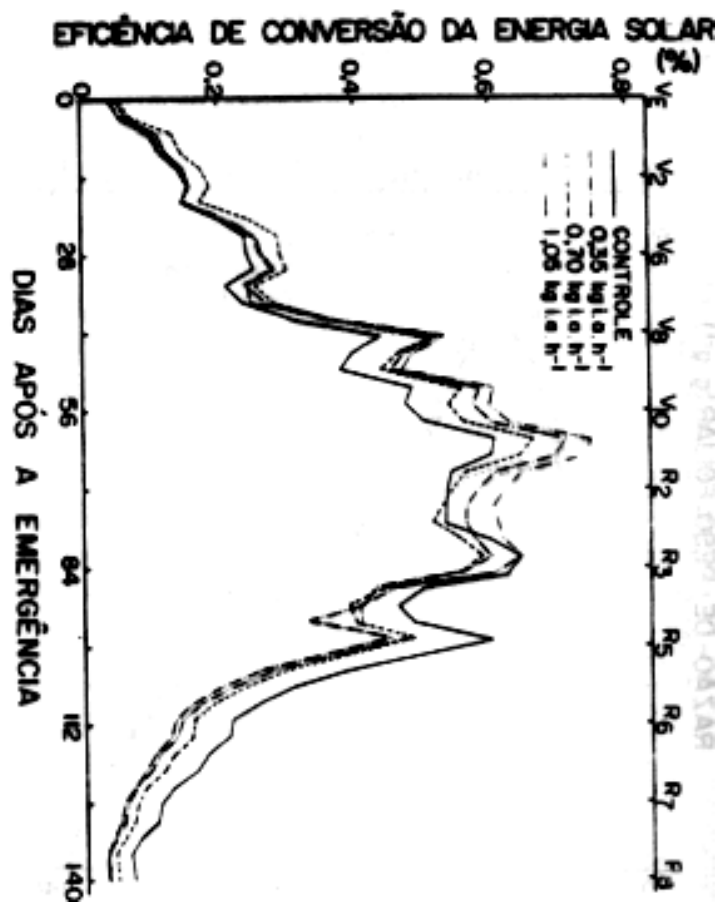

Figura 11. Eficiência de conversão da energia solar deplan tas de soja submetidas à outras dose $\mathrm{s}$ de metribuzin, sendo $V_{E}$ até R8 os estádios de des envolvimento da soja.

fected by plant population and fertilizer. Can.J. Plant Sci., 49: 675.684, 1969.

3. Clawson, K.L.; Specht, J.E. \& Blad, B.L. Growth analysis of soybean isolines differing in pubescence density. Agron. J., 78: $164-172$, 1986.

4. Dornhoff, G.M. \& Shibles, R.M. Varietal differences in net photosynthesis of soybean leaves. Crop Sci., 10: 42$45,1970$.

5. Durigan, J.C. Matocompetiçãoe comportamento de baixas doses de herbicidas na cultura da soja (Glycine max (L.) 
Merrill. Piracicaba, ESALQ, 1983. 163p. (Tese D.S.).

6. Fehr, W.R. \& Caviness, C. E. Stage of soybean development. Special Report 80 . Co-operative Extension Service. Iowa State University, Ames, Iowa, 1977, 11p.

7. Geddes, R.H.; Scott, H.D. \& oliver, L.R. Growth and wa ter use by common cocklebur (Xanthium pensylvanicum) and soybeans (Glycine max) under field conditions. Weed Sci., 27: 206-212, 1979.

8. Hagood Jr. E.S.; Bauman,T.T.; Williams Jr., J.L. \& Schrei ber, M.M. Growth analysis of soybeans (Glycine max) in competition with velvet leaf (Abutilon theophrasti).

Weed Sci., 28: 729-734, 1980

9. Hagood Jr., E.S.; Bauman, T.T; Williams Jr., J.L. \& Schrei ber, M.M. Growth analysis of soybeans (Glycine max) in competition with jimsonweed (Datura stramonium). Weed Sci., 29: 500-504, 1981.

10. Hardcastle, W.S. Differences in the tolerance of metribu$z$ in by varieties of soybeans. Weed Res., 14; 181-184, 1974 .

11. Hardcastle, W.S. Differences in susceptibility of soybean cultivars to metribuzin. Pesticide Sci., 6: 589-594, 1975. In: Weed Abstracts, 25(10): 318, 1976 . (Abstract, 2877).

12. K甘ller, H.R.; Nyquist, W.E. \& Chorush, I.S. Growth anali sys of the soybean communi ty. Crop Sci., 10: 407-41 1970.

13. Melges, E. Crescimento, conversão da energia solar e no dulação da soja (Glycine max (L.) Merrill) sob quatro ni veis de radiação solar, em
Viçosa, Minas Gerais, UFV, 1983. 78p. (Tese M.S.).

14. Oliver, L.R. Influence of soy bean (Glycine max) planting date on velvetleat (Abuti lon theophrasti) competition. Weed Sci., 27: 183-188, 1979 ,

15. Oliver, L.R.; Frans, R.E.; Tal bert, R.E. Field competi= tion between tall morningglory and soybean. I. Growth snalysis. Weed Sci., 24: $482-488,1976$.

16. Pitelli, R.A. \& Neves, A.S. Efeitos da competiçáo das plantas daninhas sobre algumas características morfológicas e agronômicas de plantas de soja. In: SEMINA RIO BRASILEIRO DE HERBICI = DAS E ERVAS DANINHAS, 12 ., Fortaleza, 1978. Resumos... Fortaleza, SBHED, 1978 . p. 104.

17. RADFORD, R.J. Growth analysis formula - Their use and abu se. Crop Sci., 7: 171-175, 1967.

18. Richards, F.J. The quantitati ve analysis of growth. In: St eward, F.C. (ed.). Plant Physiology: A Treatise. New York, Academic Press, 1969. p. 3-76.

19. Rocha, H.M.; Alvim, R. de T.; Dobereiner, J. Inf luência da intensidade de radiaçāo solar sobre o crescimento e a fixaçāo simbiótica de nitro gênio pela soja (Glycine $\max )$. Turrialba, 20: 293$298,1970$.

20. Scott, H.D. \& Batchelor, J.T. Dry weight and leaf area production rates of irriga ted determinate soybeans. Agron. J., 71: 776-782, 1979.

21. Schibles, R.M. \& Weber, C.R. Interception of solar ra- 
diation and dry matter production by various soybeans planting patterns. Crop Sci. 6: $55-59,1966$.

22. Silva Neto, P.J.; N.F. Lopes; J.F. Silva \& M.A. Oliva. Efeito do metribuzin sobre a fotossintese a tolerância em plantas de soja (Glycine max (L.) Merril1). P1anta Daminha (no prelo).

23. Silva Neto, P.J.; N.F. Lopes; J.F. Silva; M.A. Oliva \& L. R. Ferreira. Efeito do me- tribuzin no controle das plantas daninhas e na produ ção de grãos em Glycine max (L.) Merrill. Plate Daninha (no prelo).

24. Tanner, J.W. \& Ahmed, S. Growth analysis of soybeans treated with TIBA. Crop Sei., 14: 371 - 374, 1974. 